\title{
HORIZONTAL CURVATURES AND CLASSIFICATION RESULTS
}

\author{
Chiara Guidi and Vittorio Martino \\ Università di Bologna, Dipartimento di Matematica \\ Piazza di Porta S.Donato 5, 40126 Bologna, Italy; chiara.guidi12@unibo.it \\ Università di Bologna, Dipartimento di Matematica \\ Piazza di Porta S.Donato 5, 40126 Bologna, Italy; vittorio.martino3@unibo.it
}

\begin{abstract}
In this paper we consider the horizontal high-order curvatures of a real hypersurface in a generic Kähler manifold and we prove a rigidity result under a suitable assumption of parallelism. As an application we get a classification result for hypersurfaces in a complex space form.
\end{abstract}

\section{Introduction and statement of the result}

Given a complex space form $K(c)$ of constant holomorphic sectional curvature $4 c \neq 0$, it is known that do not exist real hypersurfaces with parallel second fundamental form nor umbilical real hypersurfaces (see for instance [16, Theorem 1.5] and the references therein). A weaker notion of parallelism of the second fundamental form was introduce by Kimura and Maeda in [8] for the case of the complex projective space $\mathbf{C} P^{n}$. Their definition can be easily extended to any real hypersurface $M$ in a generic Kähler manifold $K$. We say that the second fundamental form $h$ of a hypersurface $M \subseteq K$ is $\eta$-parallel if

$$
\left(\nabla_{X} h\right)(Y, Z)=0 \quad \text { for all } X, Y, Z \in T M \cap J(T M) .
$$

Here $\nabla$ and $J$ denote respectively the Levi-Civita connection and the complex structure of $K$; we refer the reader to the next section for all the precise definitions.

This notion has been intensively investigated in the case of complex space forms, also under several additional assumptions $([6,9,10,11])$; in particular, there are classification results for $\eta$-parallel hypersurfaces of Hopf type (see next section).

In this paper we restrict the second fundamental form of $M$ to the horizontal distribution $T M \cap J(T M)$, namely we denote by $h^{H}$ the horizontal part of the second fundamental form and we consider the horizontal high-order curvatures $\sigma_{k}\left(h^{H}\right)$, which are the normalized $k$-th elementary symmetric functions of the eigenvalues of $h^{H}$.

Our main theorem is a rigidity result in a generic Kähler manifold by using essentially the $\eta$-parallel condition and that one of the curvatures $\sigma_{k}\left(h^{H}\right)$ is constant. We can state it in the following way:

Theorem 1.1. Let $n \geq 1$ and $K$ be a Kähler manifold of real dimension $2 n+2$ and let $M$ be a smooth real orientable, connected and strictly $H$-convex hypersurface in $K$. Let us suppose that the second fundamental form of $M$ is $\eta$-parallel and that exists $k \in\{1, \ldots, 2 n\}$ such that $\sigma_{k}\left(h^{H}(M)\right)$ is constant. Then $M$ is a Hopf hypersurface; moreover all the eigenvalues of $h^{H}$ are constant.

https://doi.org/10.5186/aasfm.2020.4541

2010 Mathematics Subject Classification: Primary 32V40; Secondary 53C55.

Key words: $\eta$-umbilical hypersurfaces, complex space forms, $H$-convexity. 
Here, by strictly $H$-convex, we mean that $h^{H}$ is strictly positive definite as a quadratic form; we will see that this assumption cannot be dropped by showing a counterexample.

Since Hopf hypersurfaces with $\eta$-parallel second fundamental form in $K(c)$ are classified, we obtain as application of Theorem 1.1, a classification result for hypersurfaces embedded in complex space forms (Corollary 3.1); in other words we recover the property of being Hopf from the assumptions on the horizontal curvatures.

We want to mention that an analogous result has been proved in [12] where it is considered a hypersurface embedded in complex space form with two additional conditions: one is an hypothesis of parallelism (called $H$-parallel condition) and one regarding the high-order Levi curvatures, which are the normalized $k$-th elementary symmetric functions of the eigenvalues of the Levi form. In the present paper we relaxed the condition on the ambient manifold and we were able to prove our result in a generic Kähler manifold: however we remark that the two hypotheses of parallelism and the two notions of curvatures look similar but they are different, even if one specializes to the case of complex space forms.

For other similar results we refer the reader to the paper [14] and its recent generalization [3]. Moreover, for somehow related results, carried out in some cases outside the class of complex space forms, we refer to $[2,4]$.

Finally, as we said in the beginning, also umbilical hypersurfaces cannot occur in a complex space form, therefore less strong conditions have been introduced and studied also in this situation. We found classification results for $\eta$-umbilical or totally $\eta$-umbilical hypersurfaces in the case of complex space forms with $c \neq 0([6,9,17])$ : in these papers the condition $c \neq 0$ is only used to apply early classification results. Since we did not find explicit computations in the case $c=0$, in the Appendix we consider $\eta$-umbilical hypersurfaces in the flat space $\mathbf{C}^{n+1}, n>1$, obtaining an horizontal version of the classical Darboux Theorem; in particular in Example 4.1 we show that the assumption $n>1$ cannot be removed.

We would like to thank the anonymous referees for their useful comments and suggestions and for having brought to our attention the references $[2,4,17]$.

\section{Definitions and notations}

Let $K$ be a Kähler manifold of real dimension $2 n+2$ and $\omega$ be its fundamental symplectic 2 -form. We denote by $J$ the complex structure, by $g$ the Riemannian metric and by $\nabla$ its Levi-Civita connection. We recall that $g$ and $J$ are related by the following identity

$$
\omega(X, Y)=g(X, J Y) \text { for every pair of vector fields } X, Y \in T K .
$$

Moreover, both $g$ and $\nabla$ are compatible with the complex structure $J$ in the following sense

$$
J \nabla=\nabla J, \quad g(\cdot, \cdot)=g(J \cdot, J \cdot) .
$$

We consider a smooth real orientable and connected embedded manifold $M \subseteq K$ of codimension 1 on $K$, with induced metric and connection denoted again by $g$ and $\nabla$. We denote by $N$ a local choice of the unit normal to $M$ and by $X_{0}=J N$ the characteristic (or structure) vector field. Then, if $X \in T M$, one can define an endomorphism $\varphi$ of $T M$ by

$$
J X=\varphi X-\eta(X) N
$$

where $\eta$ is the one-form such that $\eta(X)=g\left(X, X_{0}\right)$. 
The horizontal distribution $H M$ is the $2 n$-dimensional subspace of $T M$ which is invariant under the action of $J$, namely

$$
H M=T M \cap J(T M)=\operatorname{ker}(\eta) .
$$

Then, the complex structure $J$ induces the following orthogonal splitting of $T M$

$$
T M=\mathbf{R} X_{0} \oplus_{g} H M .
$$

We denote the Weingarten or shape operator by

$$
A: T M \rightarrow T M, \quad A X=-\nabla_{X} N
$$

and the second fundamental form of $M$ by

$$
h(\cdot, \cdot)=g(\cdot, A \cdot) .
$$

We also need to define the horizontal part of the second fundamental form $h^{H}$ :

$$
h^{H}(X, Y)=h(X, Y) \text { for every } X, Y \in H M .
$$

Now let $\lambda_{1}, \ldots, \lambda_{2 n}$ be the eigenvalues of $h^{H}$. The $k$-th elementary symmetric function of $h^{H}$ is

$$
S_{k}\left(h^{H}\right)=\frac{1}{k !} \sum_{\substack{i_{1} \ldots, i_{k} \in \mathcal{A}_{2 n} \\ i_{1} \neq \cdots \neq i_{k}}} \lambda_{i_{1}} \ldots \lambda_{i_{k}}, \quad \text { if } k \in\{1, \ldots, 2 n\}, \quad S_{0}=1,
$$

where $\mathcal{A}_{2 n}$ is the set of indexes $\mathcal{A}_{2 n}=\{1, \ldots, 2 n\}$. We will also denote by

$$
S_{k, \hat{q}}\left(h^{H}\right)=\frac{1}{k !} \sum_{\substack{i_{1}, \ldots, i_{k} \in \mathcal{A}_{2 n} \backslash\{q\} \\ i_{1} \neq \cdots \neq i_{k}}} \lambda_{i_{1}} \ldots \lambda_{i_{k}}, \quad k, q \in\{1, \ldots, 2 n\},
$$

the $k$-th elementary symmetric function of $\lambda_{1}, \ldots \hat{\lambda}_{q}, \ldots, \lambda_{2 n}$. Hereafter we use the notation $\hat{\lambda}_{q}$ to highlight that the variable $\lambda_{q}$ is missing.

The $k$-th horizontal curvature $\sigma_{k}\left(h^{H}\right)$ of $M$ is defined as

$$
\sigma_{k}\left(h^{H}\right)=\frac{1}{\left(\begin{array}{c}
2 n \\
k
\end{array}\right)} S_{k}\left(h^{H}\right), \quad k \in\{1, \ldots, 2 n\} .
$$

These kind of horizontal curvatures appear quite naturally and have been recently studied to obtain integral formulas of Minkowski type $[13,5]$.

Let us recall also the following definitions.

Definition 2.1. A real hypersurface $M$ in a Kähler manifold $K$ is said to be strictly H-convex if $h^{H}$ is strictly positive definite as a quadratic form on $H M$.

Definition 2.2. A real hypersurface $M$ in a Kähler manifold $K$ is said to be a Hopf hypersurface if the structure vector field $X_{0}=J N$ is an eigenvector for the shape operator $A$.

Definition 2.3. A real hypersurface $M$ in a Kähler manifold $K$ is said to have $\eta$-parallel second fundamental form $h$ if

$$
\left(\nabla_{X} h\right)(Y, Z)=0 \quad \text { for every } X, Y, Z \in H M .
$$

Here we have denoted the Bortolotti derivative by $\left(\nabla_{X} h\right)(Y, Z)$ :

$$
\left(\nabla_{X} h\right)(Y, Z)=X(h(Y, Z))-h\left(\nabla_{X} Y, Z\right)-h\left(Y, \nabla_{X} Z\right) .
$$


Let us conclude the section by recalling the Codazzi equations, which we will use in the sequel. Let $K(c)$ be a complex space form, the Codazzi equations read as (see [16])

$$
\left(\nabla_{X} A\right) Y-\left(\nabla_{Y} A\right) X=-c\left(g\left(X, X_{0}\right) \varphi Y-g\left(Y, X_{0}\right) \varphi X+2 g(X, \varphi Y) X_{0}\right),
$$

for any $X, Y \in T M$. In particular we have:

$$
\left(\nabla_{X} h\right)(Y, Z)=\left(\nabla_{Y} h\right)(X, Z), \quad \text { for any } X, Y, Z \in H M \text {, }
$$

since the characteristic direction $X_{0}$ is orthogonal to $H M$.

\section{Proof of main result}

Let $\mathcal{B}=\left\{X_{0}, X_{1}, \ldots, X_{2 n}\right\}$, with $J X_{0}=-N$ be an orthonormal basis for $T M$, we define $B$ the matrix associated to the operator $J$ with respect to the basis $\mathcal{B}$

$$
(B)_{i j}=B_{i}^{j}=g\left(J X_{i}, X_{j}\right), \quad i, j \in\{1, \ldots, 2 n\} .
$$

Therefore, for $i, j \in\{1, \ldots, 2 n\}$, we have

$$
J X_{i}=\sum_{j=1}^{2 n} B_{i}^{j} X_{j}
$$

and from the compatibility relation (1) we deduce that the matrix $B$ is skew symmetric:

$$
B_{i}^{j}=g\left(J X_{i}, X_{j}\right)=-g\left(X_{i}, J X_{j}\right)=-B_{i}^{j} .
$$

We will need the following lemma:

Lemma 3.1. Let the basis $\mathcal{B}$ and the matrix $B$ be defined as above, then

$$
g\left(\left[X_{i}, J X_{i}\right], X_{0}\right)=h_{i i}+\sum_{k, l=1}^{2 n} B_{i}^{l} B_{i}^{k} g\left(-\nabla_{X_{l}} N, X_{k}\right) .
$$

Proof. We use the compatibility relations (1) to compute

$$
\begin{aligned}
g\left(\left[X_{i}, J X_{i}\right], X_{0}\right) & =g\left(\nabla_{X_{i}} J X_{i}-\nabla_{J X_{i}} X_{i}, X_{0}\right)=g\left(\nabla_{X_{i}} X_{i}, N\right)+g\left(\nabla_{J X_{i}} J X_{i}, N\right) \\
& =h_{i i}+g\left(-\nabla_{J X_{i}} N, J X_{i}\right)=h_{i i}+\sum_{j, l=1}^{2 n} g\left(-\nabla_{B_{i}^{j} X_{j}} N, B_{i}^{l} X_{l}\right) \\
& =h_{i i}+\sum_{j, l=1}^{2 n} B_{i}^{j} B_{i}^{l} g\left(-\nabla_{X_{j}} N, X_{l}\right)
\end{aligned}
$$

Now we can prove our main result.

Proof of Theorem 1.1. In order to simplify the computations, it will be convenient to choose a basis $\mathcal{B}=\left\{X_{\alpha}\right\}_{\alpha=0,1, \ldots, 2 n}$ that makes $h^{H}$ diagonal. With this choice of $\mathcal{B}$, we have that $\lambda_{i}=h_{i i}$ are the eigenvalues of $h^{H}$, for every $i=\{1, \ldots, 2 n\}$. In this basis the connection coefficients $\Gamma_{i 0}^{j}$ can be written as

$$
\Gamma_{i 0}^{j}=g\left(\nabla_{X_{i}} X_{0}, X_{j}\right)=g\left(-\nabla_{X_{i}} N, J X_{j}\right)=\sum_{l=1}^{2 n} B_{j}^{l} h_{i l}=B_{j}^{i} h_{i i} .
$$

So, the $\eta$-parallel condition (Definition 2.3)

$$
X_{l}\left(h_{j j}\right)-2 \sum_{\alpha=0}^{2 n} \Gamma_{l j}^{\alpha} h_{\alpha j}=0 \quad \text { for every } l, j \in\{1, \ldots, 2 n\}
$$


reads as

$$
X_{l}\left(h_{j j}\right)=2 \Gamma_{l j}^{0} h_{0 j}=-2 B_{j}^{l} h_{l l} h_{0 j} .
$$

Then, for every $k, l \in\{1, \ldots, 2 n\}$ we have

$$
\begin{aligned}
k ! X_{l}\left(S_{k}\left(h^{H}\right)\right) & =\sum_{\substack{i_{1}, \ldots, i_{i} \in \mathcal{A}_{2 n} \\
i_{1} \neq \cdots \neq i_{k}}} \sum_{r=1}^{k} X_{l}\left(\lambda_{i_{r}}\right) \lambda_{i_{1}} \ldots \hat{\lambda}_{i_{r}} \ldots \lambda_{i_{k}} \\
& \stackrel{(3)}{=}-2 \lambda_{l} \sum_{\substack{i_{1}, \ldots, i_{k} \in \mathcal{A}_{2 n} \\
i_{1} \neq \cdots \neq i_{k}}} \sum_{r=1}^{k} B_{i_{r}}^{l} h_{0 i_{r}} \lambda_{i_{1}} \ldots \hat{\lambda}_{i_{r}} \ldots \lambda_{i_{k}} \\
& =-2 \lambda_{l} \sum_{q=1}^{2 n} B_{q}^{l} h_{0 q} \sum_{\substack{i_{1}, \ldots \hat{q}, \ldots, i_{k} \in \mathcal{A}_{2 n} \backslash\{q\} \\
i_{1} \neq \cdots \neq i_{k}}} \lambda_{i_{1}} \ldots \hat{\lambda}_{q} \ldots \lambda_{i_{k}} \\
& =2 \lambda_{l}(k-1) ! \sum_{q=1}^{2 n} B_{l}^{q} h_{0 q} S_{k-1, \hat{q}}\left(h^{H}\right) .
\end{aligned}
$$

In the last equality it has been used the skew symmetry of the matrix $B$. Now, by hypotheses there exists $k \in\{1, \ldots, 2 n\}$ such that $S_{k}\left(h^{H}\right)$ is a positive constant, hence for this particular value of $k$ the left hand side of the chain of equalities (4) is zero. So, from the strict $H$-convexity hypothesis on $M$, we deduce

$$
\sum_{q=1}^{2 n} B_{l}^{q} h_{0 q} S_{k-1, \hat{q}}\left(h^{H}\right)=0 \quad \text { for every } l \in\{1, \ldots, n\} .
$$

Let us denote by $\gamma$ the vector $\gamma=\left(h_{01}, \ldots, h_{02 n}\right)$, and by $M_{k-1}$ the $2 n \times 2 n$ diagonal matrix with entries $\left(M_{k-1}\right)_{q q}=S_{k-1, \hat{q}}\left(h^{H}\right)$. Then, we recognize that (5) can be written in a compact form as

$$
B M_{k-1} \gamma=0
$$

Now, the strict $H$-convexity ensures the invertibility of the matrix $M_{k-1}$ and we have $B^{2}=-\operatorname{Id}_{2 n}$, then equality above implies $\gamma=0$, i.e. $M$ is a Hopf hypersurface. It remains to prove that all the eigenvalues of $h^{H}$ are constant. Since we have just shown that $\gamma=0$, equation (3) reads as

$$
X_{l}\left(\lambda_{j}\right)=0 \text { for every } l, j \in\{1, \ldots, 2 n\} .
$$

Moreover by (7) and Lemma 3.1 we have

$$
\begin{aligned}
{\left[X_{i}, J X_{i}\right]\left(\lambda_{j}\right) } & =\sum_{l=1}^{2 n} g\left(\left[X_{i}, J X_{i}\right], X_{l}\right) X_{l}\left(\lambda_{j}\right)+g\left(\left[X_{i}, J X_{i}\right], X_{0}\right) X_{0}\left(\lambda_{j}\right) \\
& \stackrel{(7)}{=} X_{0}\left(\lambda_{j}\right) g\left(\left[X_{i}, J X_{i}\right], X_{0}\right) \\
& =X_{0}\left(\lambda_{j}\right)\left(h_{i i}+\sum_{k, l=1}^{2 n} B_{i}^{l} B_{i}^{k} g\left(-\nabla_{X_{l}} N, X_{k}\right)\right) \\
& =X_{0}\left(\lambda_{j}\right)\left(h_{i i}+\sum_{l=1}^{2 n}\left(B_{i}^{l}\right)^{2} h_{l l}\right) .
\end{aligned}
$$


On the other hand, using again (7) we have

$$
\left[X_{l}, J X_{l}\right]\left(\lambda_{j}\right)=X_{j}\left(J X_{l}\left(\lambda_{j}\right)\right)-J X_{l}\left(X_{l}\left(\lambda_{j}\right)\right)=0,
$$

for every $l, j \in\{1, \ldots, 2 n\}$, so that

$$
0=\left[X_{i}, J X_{i}\right]\left(\lambda_{j}\right)=X_{0}\left(\lambda_{j}\right)\left(h_{i i}+\sum_{l=1}^{2 n}\left(B_{i}^{l}\right)^{2} h_{l l}\right)
$$

and the strict H-convexity hypotheses on $M$ implies

$$
X_{0}\left(\lambda_{j}\right)=0 \quad \text { for every } j \in\{1, \ldots, 2 n\} .
$$

This and (7) prove that $\lambda_{j}$ are constant for $j \in\{1, \ldots, 2 n\}$.

As a Corollary, we deduce a classification result for hypersurfaces embedded in complex space forms $K(c)$ and satisfying hypotheses of Theorem 1.1. It is well known that, according to the sign of the sectional curvature, zero, positive or negative, the models for this type of spaces are respectively the standard complex space $\mathbf{C}^{n+1}$ endowed with the standard Hermitian metric, the complex projective space $\mathbf{C} P^{n+1}$ with the Fubini study metric, and the complex hyperbolic space $\mathbf{C} H^{n+1}$ with the Bergman metric. In these model spaces, Hopf hypersurfaces with constant principal curvatures are classified according the "Takagi's list" and "Montiel's list" (see $[16,19,20,21,7,15,1])$. These classifications concern the case of non-vanishing sectional curvature $c$; anyway we can easily recover also the flat case. So, in view of these results, and taking into account our convexity assumption on $M$, we have the following

Corollary 3.1. Let $M$ be a real strictly H-convex hypersurface in a complex space form $K(c)$ with $\eta$-parallel second fundamental form. If $\sigma_{k}\left(h^{H}(M)\right)$ is constant for some $k \in\{1, \ldots, 2 n\}$, then

- if $c=0, M$ is contained in a sphere;

- if $c>0, M$ is contained in a type $A$ or a type $B$ hypersurface of Takagi's list;

- if $c<0, M$ is contained in a type $A$ or a type $B$ hypersurface of Montiel's list.

Proof. By Theorem 1.1 we know that $M$ is Hopf, so for $c \neq 0$ the result readly follows from the classification of Hopf hypersurfaces with $\eta$-parallel second fundamental form (see for a survey [16, Theorem 5.3]). For $c=0$ and $h_{00}$ constant the result follows from the classical classification by Segre [18] and the strict H-convexity hypothesis on $M$. Let us point out that for a general Hopf hypersurface in $\mathbf{C}^{n}, h_{00}$ might not be constant, but this is always true under the strictly $\mathrm{H}$-convexity assumption. Indeed, using Codazzi equations (2) and the fact that $M$ is Hopf one shows that $X_{i}\left(h_{00}\right)=0$ for every $i \in\{1, \ldots, 2 n\}$, and then one has $X_{0}\left(h_{00}\right)=0$, proceeding exactly as in (8) (i.e. computing the commutators and exploiting the convexity hypotheses).

We remark that the strict $H$-convexity hypothesis can not be dropped, as the following example shows.

Example 3.1. Let $\left(z_{1}, z_{2}\right)=\left(x_{1}+i y_{1}, x_{2}+i y_{2}\right) \in \mathbf{C}^{2} \simeq \mathbf{R}^{4}$. For $a, b \in \mathbf{R}$ such that $a^{2}+b^{2}=1$ we consider the domain $\Omega_{a b} \subseteq \mathbf{C}^{2}$ defined by

$$
\Omega_{a b}=\left\{\left(x_{1}, y_{1}, x_{2}, y_{2}\right) \in \mathbf{R}^{4} \simeq C^{2}: f\left(x_{1}, y_{1}, x_{2}, y_{2}\right)=\frac{x_{1}^{2}+\left(a y_{1}+b x_{2}\right)^{2}-1}{2}<0\right\}
$$


and the hypersurface $M_{a b}=\partial \Omega_{a b}$. Let $r=a y_{1}+b x_{2}$, then the norm of the Euclidean gradient of $f$ is $|D f|=\sqrt{x_{1}^{2}+r^{2}\left(a^{2}+b^{2}\right)}=1$. The unit inward normal to $M$ is given by

$$
N=-x_{1} \partial_{x_{1}}-r a \partial_{y_{1}}-r b \partial_{x_{2}},
$$

and we consider $\mathcal{B}=\left\{X_{0}, X, Y\right\}$ the basis for $T M$ given by

$$
\begin{aligned}
X_{0}=J(N) & =r a \partial_{x_{1}}-x_{1} \partial_{y_{1}}-r b \partial_{y_{2}}, \\
X & =r b \partial_{x_{1}}-x_{1} \partial_{x_{2}}+r a \partial_{y_{2}}, \\
Y=J(X) & =r b \partial_{y_{1}}-r a \partial_{x_{2}}-x_{1} \partial_{y_{2}} .
\end{aligned}
$$

We compute the second fundamental form with respect to the basis $\mathcal{B}$ :

$$
h(X, X)=r b X\left(x_{1}\right)-x_{1} X(r b)=b^{2},
$$

and similarly

$$
h(Y, Y)=0, \quad h(X, Y)=0, \quad h\left(X_{0}, X_{0}\right)=a^{2}, \quad h\left(X_{0}, X\right)=a b, \quad h\left(X_{0}, Y\right)=0 .
$$

From which we deduce that $M$ is $\mathrm{H}$-convex (but not strictly $\mathrm{H}$-convex), it has constant horizontal curvatures, but it is not Hopf if $a, b \neq 0$.

\section{Appendix}

We recall that a hypersurface is called umbilical if there exists a scalar function $f$ such that the second fundamental form reads as $h=f g$, being $g$ the metric. A real hypersurface in a Kähler manifold is said $\eta$-umbilical if there exists a scalar function $L$ such that

$$
h(X, Y)=L g(X, Y) \text { for every } X, Y \in H M .
$$

This condition has been studied in literature, in particular a slightly stronger condition has been often considered, namely a totally $\eta$-umbilical assumption, which basically means that the hypersurface is $\eta$-umbilical and Hopf. We found some results in this regard (see for instance $[6,9,17]$ and the references therein), but all of them concern the case of complex space forms $K(c)$ with $c \neq 0$. Here we show a rigidity result in $\mathbf{C}^{n+1}$ that is analogue to the classical Darboux Theorem in the standard Euclidean space: of course the proof is easier than the case with $c \neq 0$. We have the following

Theorem 4.1. Let $n>1$ and let $M$ be a smooth real orientable and connected $\eta$-umbilical hypersurface in $\mathbf{C}^{n+1}$. Then $M$ is part of a sphere or a ruled hypersurface of the type $\mathbf{C}^{n} \times \gamma$ with $\gamma$ a curve in $\mathbf{C}^{n+1}$ such that $\dot{\gamma}=X_{0}$.

Proof. We consider $\mathcal{B}=\left\{X_{0}, X_{1}, \ldots, X_{2 n}\right\}$ an orthonormal basis of $T M$ such that $X_{n+s}=J X_{s}$ for every $s \in\{1, \ldots, n\}$ and $X_{0}=J N$. As a convention, we will use Latin letters to denote indices running from 1 to $2 n$ (i.e. purely horizontal) and Greek letters to denote indices running from 0 to $2 n$ (i.e. involving also the characteristic direction), in particular we will use the letters $s, t$ to denote indexes running from 1 to $n$.

Since $M$ is $\eta$-umbilical, for a suitable function $L$, we have

$$
h_{i j}=h\left(X_{i}, X_{j}\right)=L g\left(X_{i}, X_{j}\right)=\delta_{i j} L, \quad \text { for every } i, j \in\{1, \ldots, 2 n\} .
$$

We want to show that $L$ is constant on $M$. We start by writing some relations involving $\Gamma_{\alpha \beta}^{\gamma}=g\left(\nabla_{X_{\alpha}} X_{\beta}, X_{\gamma}\right)$ with $\alpha, \beta, \gamma \in\{0, \ldots, 2 n\}$ which are the coefficients 
of the connection with respect to the basis $\mathcal{B}$. Since $\mathcal{B}$ is orthonormal we have

$$
\Gamma_{\alpha \beta}^{\beta}=0 \quad \text { and } \quad \Gamma_{\alpha \beta}^{\gamma}=-\Gamma_{\alpha \gamma}^{\beta} .
$$

Moreover exploiting the relations $X_{n+s}=J X_{s}$ for every $s \in\{1, \ldots, n\}$ and the compatibility relations (1) we have

$$
\begin{aligned}
\Gamma_{s n+t}^{0} & =g\left(\nabla_{X_{s}} X_{n+t}, X_{0}\right)=g\left(-\nabla_{X_{s}} X_{t},-N\right)=h_{s t}, \\
\Gamma_{n+t s}^{0} & =g\left(\nabla_{X_{n+t}} X_{s}, X_{0}\right)=g\left(\nabla_{X_{n+t}} X_{n+s},-N\right)=-h_{n+t n+s}, \\
\Gamma_{s s}^{0} & =g\left(\nabla_{X s} X_{s}, X_{0}\right)=g\left(\nabla_{X_{s}} X_{n+s},-N\right)=-h_{s n+s}, \\
\Gamma_{n+t n+t}^{0} & =g\left(\nabla_{X_{n+t}} X_{n+t}, X_{0}\right)=g\left(-\nabla_{X_{n+t}} X_{t},-N\right)=h_{n+t t} .
\end{aligned}
$$

for every $s, t \in\{1, \ldots, n\}$. Then, from the $\eta$-umbilical hypotheses (9) we get

$$
\Gamma_{s n+t}^{0}=\delta_{s t} L, \quad \Gamma_{n+t s}^{0}=-\delta_{s t} L, \quad \Gamma_{s, s}^{0}=\Gamma_{n+t n+t}^{0}=0 .
$$

Now we use Codazzi equation (2) and the relations (10) and (11), involving the connection coefficients, to prove that

$$
\begin{aligned}
X_{s}(L) & =X_{s}\left(h_{n+t n+t}\right)=3 \delta_{s t} h_{0 n+t} L \\
X_{n+s}(L) & =X_{n+s}\left(h_{t t}\right)=-3 \delta_{s t} h_{0 t} L \quad \text { for every } s, t \in\{1, \ldots, n\} .
\end{aligned}
$$

We perform the computations only for the case $X_{s}(L)$, since the case $X_{n+s}(L)$ is similar:

$$
\begin{aligned}
& X_{s}(L)=X_{s}\left(h_{n+t n+t}\right)=X_{s}\left(h_{n+t n+t}\right)-X_{n+t}\left(h_{s n+t}\right) . \\
& \stackrel{(2)}{=} \sum_{\alpha=0}^{2 n}\left(2 \Gamma_{s n+t}^{\alpha} h_{\alpha n+t}-\Gamma_{n+t s}^{\alpha} h_{\alpha n+t}-\Gamma_{n+t n+t}^{\alpha} h_{s \alpha}\right) \\
& \stackrel{(9)}{=} 2 \Gamma_{s n+t}^{0} h_{0 n+t}-\Gamma_{n+t s}^{0} h_{0 n+t}-\Gamma_{n+t n+t}^{0} h_{s 0} \\
& +\sum_{i=1}^{2 n}\left(2 \Gamma_{s n+t}^{i} \delta_{i n+t}-\Gamma_{n+t s}^{i} \delta_{i n+t}-\Gamma_{n+t n+t}^{i} \delta_{s i}\right) L \\
& =2 \Gamma_{s n+t}^{0} h_{0 n+t}-\Gamma_{n+t s}^{0} h_{0 n+t}-\Gamma_{n+t n+t}^{0} h_{s 0}+ \\
& +\left(2 \Gamma_{s n+t}^{n+t}-\Gamma_{n+t s}^{n+t}-\Gamma_{n+t n+t}^{s}\right) L \\
& \stackrel{(10)}{=} 2 \Gamma_{s n+t}^{0} h_{0 n+t}-\Gamma_{n+t s}^{0} h_{0 n+t}-\Gamma_{n+t n+t}^{0} h_{s 0} \\
& \stackrel{(11)}{=} 3 \delta_{s t} h_{0 n+t} L \text {. }
\end{aligned}
$$

In particular, the identities (12) with the choice $t \neq s$ imply

$$
X_{i}(L)=0 \quad \text { for every } i \in\{1, \ldots, 2 n\}
$$

Note that the choice $t \neq s \in\{1, \ldots, n\}$ implicitly uses the assumption on the dimension $n>1$. Now we use the commutators to gain information on $X_{0}(L)$. Recalling 
that $X_{i}(L)=0$ for every $i \in\{1, \ldots, 2 n\}$ we find

$$
\begin{aligned}
{\left[X_{s}, X_{n+s}\right](L)=} & \left(\nabla_{X_{s}} X_{n+s}-\nabla_{X_{n+s}} X_{s}\right)(L) \\
= & \sum_{i=1}^{2 n} g\left(\nabla_{X_{s}} X_{n+s}-\nabla_{X_{n+s}} X_{s}, X_{i}\right) X_{i}(L) \\
& +g\left(\nabla_{X_{s}} X_{n+s}-\nabla_{X_{n+s}} X_{s}, X_{0}\right) X_{0}(L) \\
= & g\left(\nabla_{X_{s}} X_{n+s}-\nabla_{X_{n+s}} X_{s}, X_{0}\right) X_{0}(L) \\
= & \left(\Gamma_{s, n+s}^{0}-\Gamma_{n+s, s}^{0}\right) X_{0}(L) \\
& \stackrel{(11)}{=} 2 L X_{0}(L) .
\end{aligned}
$$

On the other hand,

$$
\left[X_{s}, X_{n+s}\right](L)=X_{s}\left(X_{n+s}(L)\right)-X_{n+s}\left(X_{s}(L)\right)=0
$$

so that

$$
2 L X_{0}(L)=0
$$

At this stage we are left with just two cases:

i) $L(p)=0$, for every $p \in M$.

ii) There exists $p_{0} \in M$ such that $L\left(p_{0}\right) \neq 0$.

Using the compatibility relations (1), for every $i, j \in\{1, \ldots, 2 n\}$ we have

$$
\begin{aligned}
g\left(\left[X_{i}, X_{j}\right], X_{0}\right) & =-g\left(\nabla_{X_{i}} J X_{j}-\nabla_{X_{j}} J X_{i}, N\right) \\
& =-\left(h\left(X_{i}, J X_{j}\right)-h\left(X_{j}, J X_{i}\right)\right) .
\end{aligned}
$$

So, in the first case, since $L \equiv 0$ we have $g\left(\left[X_{i}, X_{j}\right], X_{0}\right)=0$, hence the horizontal distribution $H M=\operatorname{span}\left\{X_{1}, \ldots, X_{2 n}\right\}$ is involutive and therefore, by Frobenius Theorem, $M$ is part of a ruled hypersurface of the type type $\mathbf{C}^{n} \times \gamma$ with $\gamma$ a curve in $\mathbf{C}^{n+1}$ such that $\dot{\gamma}=X_{0}$.

In the second case, we have to prove that $M$ is part of a sphere. Let us start by showing that $L$ is constant on $M$. Since $L\left(p_{0}\right) \neq 0$, by continuity, $L \neq 0$ in a neighborhood $V_{p_{0}} \subseteq M$ of $p_{0}$. Hence from (14) we deduce that $X_{0}(L)=0$ on $V_{p_{0}}$, moreover we have proved $X_{i}(L)=0$ for every $i=\{1, \ldots, 2 n\}$, then $L$ is constant on $V_{p_{0}}$. Now, from the continuity of $L$ and the connectedness of $M$ we have that $L$ is a non-vanishing constant on the whole $M$. Then, equations (12), with the choice $t=s$, imply that $h_{0 i}=0$ for every $i \in\{1, \ldots, 2 n\}$. Now, let us identify $p$ with the position vector field, namely

$$
p=\sum_{k=1}^{2 n+2} g\left(p, \partial_{x_{k}}\right) \partial_{x_{k}}=\sum_{k=1}^{2 n+2} p^{k} \partial_{x_{k}} .
$$

Let also the unit normal be

$$
N=\sum_{k=1}^{2 n+2} g\left(N, \partial_{x_{k}}\right) \partial_{x_{k}}=\sum_{k=1}^{2 n+2} N^{k} \partial_{x_{k}} .
$$


We are going to show that the functions $\varphi_{k}:=p^{k}+\frac{N^{k}}{L}$ are constant. Indeed, for every $i \in\{1, \ldots, 2 n\}$ and $k \in\{1, \ldots, 2 n+2\}$ we have

$$
\begin{aligned}
X_{i}\left(\varphi_{k}\right) & =X_{i}\left(g\left(p, \partial_{x_{k}}\right)\right)+\frac{1}{L} X_{i}\left(g\left(N, \partial_{x_{k}}\right)\right) \\
& =g\left(\nabla_{X_{i}} p, \partial_{x_{k}}\right)+g\left(p, \nabla_{X_{i}} \partial_{x_{k}}\right)-\frac{1}{L} g\left(-\nabla_{X_{i}} N, \partial_{x_{k}}\right)+\frac{1}{L} g\left(N, \nabla_{X_{i}} \partial_{x_{k}}\right) \\
& =g\left(\nabla_{X_{i}} p, \partial_{x_{k}}\right)-\frac{1}{L} g\left(-\nabla_{X_{i}} N, \partial_{x_{k}}\right) \\
& =g\left(X_{i}, \partial_{x_{k}}\right)-\frac{1}{L} g\left(L X_{i}, \partial_{x_{k}}\right)=0 .
\end{aligned}
$$

In the fourth equality we have used the identities $h\left(X_{i}, X_{j}\right)=\delta_{i j} L$ and $h_{0 i}=0$ for every $i, j \in\{1, \ldots, 2 n\}$. Then the same procedure used to obtain (14) gives $2 L X_{0}\left(\varphi_{k}\right)=$ 0 , so $X_{0}\left(\varphi_{k}\right)=0$. Consequently $\varphi_{k}$ is constant, for every $k \in\{1, \ldots, 2 n+2\}$. Therefore, for every $k$ we have $p^{k}+\frac{N^{k}}{L}=c^{k}$ for some constant $c^{k}$, hence $\|p-c\|=\frac{1}{|L|}$ where $c=\left(c^{1}, \ldots, c^{2 n+2}\right)$. This proves that $M$ is part of a sphere of center $c$ and radius $\frac{1}{|L|}$.

We explicitly notice that in the above theorem we have assumed $n>1$ and this hypothesis has been exploited to prove (13). When we consider $n=1$ the situation changes drastically. Indeed, there exist $\eta$-umbilical hypersurfaces $M \subseteq \mathbf{C}^{2}$ that are not contained in spheres nor in ruled hypersurfaces of the type $\mathbf{C}^{n} \times \gamma$. We have the following example

Example 4.1. Let $\mathbf{C}^{2} \simeq \mathbf{R}^{4}$ with coordinates $\left(z_{1}, z_{2}\right)=\left(x_{1}+i y_{1}, x_{2}+i y_{2}\right)$. For $a, b \in \mathbf{R}$ we consider the domain $\Omega_{a b}$, defined by

$$
\Omega_{a b}=\left\{\left(x_{1}, y_{1}, x_{2}, y_{2}\right) \in \mathbf{R}^{4}: f\left(x_{1}, y_{1}, x_{2}, y_{2}\right)=a \frac{x_{1}^{2}+y_{1}^{2}}{2}+b \frac{x_{2}^{2}+y_{2}^{2}}{2}-1<0\right\}
$$

and the hypersurface $M_{a b}=\partial \Omega_{a b}$. The norm of the gradient of $f$ is $|D f|=$ $\left(a^{2}\left(x_{1}^{2}+y_{1}^{2}\right)+b^{2}\left(x_{2}^{2}+y_{2}^{2}\right)\right)^{\frac{1}{2}}$, and the inward unit normal to $M$ is given by

$$
N=-\frac{1}{|D f|}\left(a x_{1} \partial_{x_{1}}+a y_{1} \partial_{y_{1}}+b x_{2} \partial_{x_{2}}+b y_{2} \partial_{y_{2}}\right)
$$

and we consider $\mathcal{B}=\left\{X_{0}, X, Y\right\}$ the basis for $T M$ given by

$$
\begin{array}{r}
X_{0}=J(N)=\frac{1}{|D f|}\left(a y_{1} \partial_{x_{1}}-a x_{1} \partial_{y_{1}}+b y_{2} \partial_{x_{2}}-b x_{2} \partial_{y_{2}}\right), \\
X=\frac{1}{|D f|}\left(b x_{2} \partial_{x_{1}}-b y_{2} \partial_{y_{1}}-a x_{1} \partial_{x_{2}}+a y_{1} \partial_{y_{2}}\right), \\
Y=J(X)=\frac{1}{|D f|}\left(b y_{2} \partial_{x_{1}}+b x_{2} \partial_{y_{1}}-a y_{1} \partial_{x_{2}}-a x_{1} \partial_{y_{2}}\right) .
\end{array}
$$

We compute the horizontal second fundamental form with respect to the basis $\mathcal{B}$ :

$$
h(X, X)=\frac{1}{|D f|^{3}}\left(b^{2} y_{2}^{2} a+b^{2} x_{2}^{2} a+a^{2} y_{1}^{2} b+a^{2} x_{1}^{2} b\right)=\frac{2 a b}{|D f|^{3}}
$$


and similarly

$$
h(Y, Y)=\frac{2 a b}{|D f|^{3}}, \quad h(X, Y)=0 .
$$

Thus $M_{a b}$ is a $\eta$-umbilical hypersurface but is not a sphere nor of the type $\mathbf{C} \times \gamma$, if $a \neq b$.

\section{References}

[1] Berndt, J.: Real hypersurfaces with constant principal curvatures in complex hyperbolic space. - J. Reine Angew. Math. 395, 1989, 132-141.

[2] Berndt, J., and Y. Suh: Contact hypersurfaces in Kähler manifolds. - Proc. Amer. Math. Soc. 143:6, 2015, 2637-2649.

[3] Cho, J. T., and M. Kimura: Levi-umbilical real hypersurfaces in a complex space form. Nagoya Math. J. 229, 2018, 99-112.

[4] Cho, J. T., and M. Kimura: Levi-umbilical real hypersurfaces in Hermitian symmetric spaces. - Topology Appl. 264, 2019, 145-157.

[5] Guidi, C., and V. MarTino: Horizontal Newton operators and high-order Minkowski formula. - Submitted.

[6] Iтон, T., and S. Maeda: Characterization of totally $\eta$-umbilic real hypersurface in nonflat complex space forms by some inequality. - Proc. Japan Acad. Ser. A Math. Sci. 80:5, 2004, $61-64$.

[7] Kimura, M.: Real hypersurfaces and complex submanifolds in complex projective space. Trans. Amer. Math. Soc. 296, 1986, 137-149.

[8] Kimura, M., and S. Maeda: On real hypersurfaces of a complex projective space. - Math. Z. 202, 1989, 299-311.

[9] Kon, M.: A characterization of totally $\eta$-umbilical real hypersurfaces and ruled real hypersurfaces of a complex space form. - Czechoslovak Math. J. 58, 2008, 1279-1287.

[10] Kon, S. H., and T. H. Loo: Real hypersurfaces in a complex space form with $\eta$-parallel shape operator. - Math. Z. 269:1-2, 2011, 47-58.

[11] Kon, S. H., and T. H. Loo: On characterizations of real hypersurfaces in a complex space form with $\eta$-parallel shape operator. - Canad. Math. Bull. 55:1, 2012, 114-126.

[12] Martino, V., and G. Tralli: High-order Levi curvatures and classification results. - Ann. Global Anal. Geom. 46, 2014, 351-359.

[13] Martino, V., and G. Tralli: On the Minkowski formula for hypersurfaces in complex space forms. - Submitted.

[14] Monti, R., and D. Morbidelli: Levi umbilical surfaces in complex space. - J. Reine Angew. Math. 603, 2007, 113-131.

[15] Montiel, S.: Real hypersurfaces of a complex hyperbolic space. - J. Math. Soc. Japan 37, $1985,515-535$.

[16] Niebergall, R., and P. J. Ryan: Real hypersurfaces in complex space forms. - In: Tight and taut submanifolds (Berkeley, CA, 1994), Math. Sci. Res. Inst. Publ. 32, Cambridge Univ. Press, 1997, 233-305.

[17] Ortega, M.: Classifications of real hypersurfaces in complex space forms by means of curvature conditions. - Bull. Belg. Math. Soc. 9:3, 2002, 351-360.

[18] Segre, B.: Famiglie di ipersuperficie isoparametriche negli spazi euclidei ad un qualunque numero di dimensioni. - Atti Accad. Naz. Lincei Rend. VI. Ser. 27, 1938, 203-207.

[19] TAKagi, R. On homogeneous real hypersurfaces in a complex projective space. - Osaka J. Math. 10, 1973, 495-506. 
[20] TAKAGi, R.: Real hypersurfaces in a complex projective space with constant principal curvatures. - J. Math. Soc. Japan 27, 1975, 43-53.

[21] TAKAGI, R.: Real hypersurfaces in a complex projective space with constant principal curvatures. II. - J. Math. Soc. Japan 27, 1975, 507-516.

Received 18 February 2019 • Accepted 24 September 2019 